\title{
The Influence of Turmeric and Curcumin on Female Reproductive Processes
}

\author{
Author \\ Alexander V. Sirotkin ${ }^{(\mathbb{D}}$

\section{Affiliation} \\ Department of Zoology and Anthropology, Constantine the \\ Philosopher University in Nitra, Nitra, Slovakia
}

Key words

curcumin, turmeric, health, ovary, proliferation, apoptosis, hormone, fecundity

$\begin{array}{ll}\text { received } & \text { February 19, } 2021 \\ \text { accepted after revision } & \text { June 22, 2021 } \\ \text { published online } & \text { August 20, 2021 }\end{array}$

Bibliography

Planta Med 2022; 88: 1020-1025

DOI $10.1055 / a-1542-8992$

ISSN 0032-0943

(c) 2021. Thieme. All rights reserved.

Georg Thieme Verlag KG, Rüdigerstraße 14,

70469 Stuttgart, Germany

Correspondence

Prof. Dr. Alexander V. Sirotkin, PhD, DrSc

Department of Zoology and Anthropology,

Constantine the Philosopher University in Nitra

Tr. A. Hlinku 1, 94974 Nitra, Slovakia

Phone: +421903561120

asirotkin@ukf.sk

\section{ABSTRACT}

The present review summarizes the available knowledge concerning the action of curcumin, the best-known polyphenol among the rhizomes of Curcumas, on female reproductive processes and their dysfunctions. Curcumin affects a number of physiological processes, including female reproduction (puberty, reproductive aging, ovarian follicullogenesis and oogenesis, and fecundity). Curcumin can affect these processes via changes in the release and reception of pituitary and ovarian hormones, growth factors and cytokines. Furthermore, it can influence the response of ovarian cells to these substances and external environmental factors. Finally, curcumin can affect oxidative processes within the ovary and numerous intracellular signalling pathways related to ovarian cell proliferation and apoptosis. These effects suggest the applicability of curcumin for stimulation of female reproductive processes in vivo and in vitro, as well as for the prevention, mitigation, and treatment of various reproductive disorders from ovarian insufficiency and infertility to polycystic ovarian syndrome and ovarian cancer.

\section{Introduction}

Curcuma/turmeric is a popular spice. Its health-promoting effects have already been described in several reviews [1-5]. On the other hand, only one publication [6] reviewed curcuma action on reproductive processes, though it was focused on cancerogenesis and not on curcuma action on a healthy reproductive system. The present publication is the first attempt at summarizing the available information concerning the action of curcuma and its constituents on a healthy female reproductive system, as well as of the data concerning their ability to prevent and to treat reproductive disorders.

A search of the literature was performed in agreement with PRISMA-SCR criteria [7]. To find related articles, Pubmed, Web of Science, and SCOPUS databases were searched for articles published between the years 2000 and 2021. In cases of repeated or conflicting information or references, the most recent source was used. Words used in the search were curcumin, turmeric, review, health, metabolism, fertility, ovarian, and mechanisms.

\section{Provenance and Properties}

The herbs that belong to the genus Curcuma (Zingiberaceae) are currently cultivated in tropical and subtropical areas of Asia, Australia, and South America. Approximately 93-100 species of Curcuma are described now. These herbs are widely used for the coloring and flavoring of food, for preparation of food additives (the most known is curry powder), dyes, perfumes, and cosmetics as well as in folk medicine. The most known and useful Curcuma species are Curcuma longa (Indian turmeric) and Curcuma zedoaria (zedoary). Turmeric is native to Southeast Asia but is now cultivated extensively worldwide. Zedoary originated from India and Indonesia but it is cultivated in many subtropical regions around 
the world. The rhizome is the most commonly used part of these plants.

Turmeric rhizome typically contains carbohydrates (69.4\%), protein $(6.3 \%)$, fat $(5.1 \%)$, and minerals $(3.5 \%)$. The main active components of the rhizome are the non-volatile curcuminoids and the volatile oil [3]. A main biologically active component of C. longa $L$. is curcumoids curcumin, which is chemically known as diferuloylmethane [1,7-bis(4-hydroxy-3-methoxyphenyl)-1,6-eptadiene-3,5-dione], and its polyphenolic derivatives and metabolites, demethoxycurcumin and bisdemethoxycurcumin [1]. The major components of curcuma oils are sesquiterpenoids and monoterpenoids $[3,4]$.

\section{Mechanisms of Action}

Curcumin has antioxidant, anti-inflammatory, antimicrobial, proapoptotic, antiproliferative, antiangiogenic, and antimutagenic activities [2,4,8-10], which determine its physiological and therapeutic effects. For example, the antioxidant action of curcumin defines its ability to prevent oxidative stress, DNA, and protein damage [10]. Common molecular intracellular targets and mediators of curcuma action could be cytokines and transcription factors promoting inflammatory processes, some protein kinases, and enzymes. Curcumin can affect a number of signaling molecules like Nrf2, $\beta$-catenin, NF- $\kappa$ B, p38 MAPK, DNA (cytosine-5)methyltransferase-1, COX-2, TNF- $\alpha, \quad$ 5-lipoxygenase, PGE2, FOXO3, generation nitric oxide and reactive oxygen species, cyclin D1, VEGF, glutathione, cytosolic PLA2, and p-Tau (p-T). For example, curcumin can suppress inflammatory processes through the downregulation of intracellular promoters of inflammation such as Nrf2, NF-kB, STAT3, reactive oxygen species, and COX-2 (see [2] for review). On the other hand, curcumin can boost the functions of immunocompetent cells, the production of inflammatory cytokines, and reparative processes [11,12]. Furthermore, curcumin can suppress cancerogenesis [10,13] and other illnesses [14] targeting microRNAs and long non-coding RNAs. The physiological action of turmeric oils could be explained by their ability to inhibit reactive oxygen species, COX-2, and other signaling molecules as well [3]. This ability of turmeric molecules to modulate many extra- and intracellular signaling pathways related to various dysfunctions and diseases suggests that it is a polyphenol with many targets and physiological and medicinal actions $[2,4]$.

\section{Physiological and Therapeutic Actions}

The current literature describes anti-inflammatory, antioxidant, anticancer, antimutagenic, antimicrobial, anti-obesity, hypolipidemic, cardioprotective, wound healing, and neuroprotective properties of curcumin $[4,5,11,15]$. Curcumin-containing Curcumas are popular in Asian folk medicine to treat pneumonia, bronchitis, leucorrhea, diarrhea, dysentery, infectious wounds or abscesses, and insect bites. In this context, curcumin is applicable as a carminative, digestive aid, stomachic, anthelmintic, appetizer, tonic, and laxative. It is also used as a treatment against fever, gastritis, dysentery, infections, hypercholesterolemia, hypertension, chest congestion, arthritis, jaundice, liver and gall bladder problems, cough, urinary tract infections, skin diseases, dia- betic wounds, and menstrual discomfort $[3,4]$. It can be a potent immunostimulator and anticancer drug [11]. Many reports indicate that curcumin can regulate blood sugar levels, decrease blood pressure, protect nerve cells, and enhance immunity. Therefore, curcumin may be especially beneficial for the elderly in the prevention and treatment of age-related disorders like diabetes and diabetic complications as well as neurodegenerative and cardiovascular diseases [5].

As mentioned above, polyphenol curcumin has antioxidant, anti-inflammatory, anticancer, antiangiogenic, wound healing, and antimicrobial properties. Due to these properties, curcumin has been shown to be efficient for the treatment of numerous chronic diseases including various types of cancers, diabetes, obesity, and cardiovascular, pulmonary, neurological, infectious, and autoimmune diseases $[2,8,9,16]$. Furthermore, curcumin can be a synergist to other plant polyphenols with therapeutic action like resveratrol, catechins, piperine, quercetin, and genistein [2].

Curcuma essential oils, like curcumin, possess a number of biological and medicinal properties, including anti-inflammatory, anticancer, antiproliferative, hypocholesterolemic, antidiabetic, antihepatotoxic, antidiarrheal, diuretic, antirheumatic, hypotensive, antioxidant, antimicrobial, antiviral, insecticidal, larvicidal, antivenomous, carminative, and antithrombotic effects. These oils can promote functions of the immune system, blood circulation, detoxication, and digestion (see $[3,4,16]$ for reviews).

Curcuma longa extracts possess renal protective, hepatoprotective, cardioprotective, antidiabetic, neuroprotective, and gonadoprotective effects $[2,4,6]$.

Moreover, curcumin can mitigate or prevent the harmful action of environmental contaminants [15]. For example, it has been shown to reduce genotoxicity, nephrotoxicity, hepatotoxicity, skin diseases, angiogenesis, reproductive toxicity, neurotoxicity, and immunotoxicity of arsenic [10]. Either no or no substantial negative curcumin side effects have been observed $[4,16]$.

\section{Effect on Female Reproductive Processes}

\section{Effect on reproductive organs}

Feeding rats curcumin did not affect weight of their ovaries or size of their litters [17]. On the other hand, an artificial pegylated curcumin analogue inhibited rat ovarian follicle development, sexual maturation, and fecundity [18]. Curcumin promoted ovarian growth, follicullogenesis [19], and mitigated the age-dependent suppression of ovarian functions in mice [20], d-galactose-induced ovarian failure in mice [21] and ovarian failure induced by cyclophosphamide in rats [22]. Dietary curcumin promoted ovarian follicullogenesis and ovulation in the fish Pseudotropheus socolofi [23]. The plant additive containing curcumin promoted ovarian follicle development in the buffalo as well [12]. On the contrary, Destici Isgoren et al. [24] did not observe an effect of the curcuma extract on rat ovarian follicle development. Feeding rabbits turmeric powder did not influence their ovarian size or weight, but it increased the number and diameter of ovarian follicles. It did not affect rabbit fecundity but increased the viability of their pups [25]. 
Tossetta et al. [15] presented evidence that curcumin can be useful for managing pregnancy complications (preeclampsia, gestational diabetes mellitus, fetal growth retardation, preterm birth, and response to toxins and pathogens).

Therefore, the data listed above suggest curcumin can both up- and downregulate puberty, ovarian follicullogenesis, and fecundity, as well as prevent complications during pregnancy. The character of its effect depends on the animal species and experimental model.

\section{Effect on ovarian cell functions}

One in vitro study showed a suppressive effect of curcumin on basic healthy ovarian cell functions. In a study by Kádasi et al. [26], the addition of curcumin reduced proliferation and viability and promoted apoptosis in cultured porcine ovarian granulosa cells. Numerous studies on ovarian cancer cells showed the ability of curcumin to inhibit the cell cycle and to promote apoptosis [8, 13, 17, 27-35].

In vivo studies have shown the opposite, stimulatory action of curcumin and its analogue on murine ovaries. In these studies, curcumin stimulated proliferation and inhibited apoptosis in murine [21,35-37] and rat [24] ovarian cells. Moreover, curcumin stimulated ovarian oogenesis in mice $[19,36,37]$. Therefore, the available publications demonstrate the curcumin has an influence on ovarian cell mitosis, meiosis, apoptosis, and viability.

\section{Effect on reproductive hormones}

In vivo experiments demonstrated the ability of curcumin to decrease the concentration of gonadotropins FSH (follicle stimulating hormone) and LH (luteinizing hormone) in rat serum [19,22]. Surprisingly, a decrease in gonadotropin release was associated with a rise in the serum level of estradiol and anti-mullerian hormones $[19,22]$, which should be upregulated by gonadotropins [38]. Rabbits fed curcumin had an increase in the ovarian production of progesterone but a decrease the ovarian release of testosterone and leptin. Moreover, feeding rabbits curcumin altered the response of ovarian cells to $\mathrm{LH}$, as LH reduced leptin release by ovarian cells of the control rabbits but increased leptin release by ovarian cells of rabbits fed curcumin [25].

In vitro experiments also showed the stimulatory influence of curcumin on progesterone and testosterone release by cultured porcine granulosa cells [26], and its inhibitory action on progesterone release by cultured luteal cells from rat ovaries [39].

Some experiments, however, did not show any curcumin influence on FSH, progesterone, or estradiol [17] or anti-mullerian hormones [24] in rat plasma. Moreover, they demonstrated a curcumin-induced increase in $\mathrm{LH}$ and a decrease in prolactin concentration in the plasma of rats fed curcumin [17]. Thus, the performed studies demonstrated that curcumin influences (1) the release of gonadotropins by pituitary cells, (2) the release of hormones by ovarian cells, and (3) the response of ovarian cells to gonadotropins.

\section{Mechanisms of action on female reproductive processes}

It can be easily noted that the curcumin effects observed in in vivo and in vitro studies are different, and sometimes contradictory.
This fact indicates the action of curcumin directly on ovarian cells and on their upstream regulators. The best-known endocrine and paracrine/autocrine regulators of reproductive and other physiological actions are hormones. It is well documented that fecundity is defined by the growth, development, and atresia of follicles in the ovary, and that these processes are under the control of gonadotropins, ovarian steroids, and peptide hormones [38]. The previous sections of this review have demonstrated that curcumin can influence pituitary and ovarian hormones, which can mediate curcumin action on reproductive processes.

Curcumin as a phytoestrogen [40] can interact with the steroid hormones and their receptors, affect the hypothalamo-hypophysial-ovarian axis, and treat some reproductive disorders [41]. For example, in mouse ovaries, curcumin can suppress androgen receptors and stimulate 3-beta-hydroxysteroid dehydrogenase [20], although it does not affect aromatase [42]. Furthermore, curcumin mitigated the action of dehydroepiandrosterone on mouse ovarian cell apoptosis [35]. Therefore, the influence of curcumin on ovarian functions and state could be mediated by its action on ovarian steroid hormone receptors.

There are indications that curcumin action on the ovary (at least the ovary suffering from polycystic ovarian syndrome) can be mediated by its suppressive action on inflammation [6] and inflammation-related cytokine interleukin-6, tumor necrosis factor$\alpha$, C-reactive protein [43], growth factors GDF-9 and BMP-15 [19, 44], and vascular endothelial growth factor [6].

Furthermore, the influence of curcumin on intracellular regulators of the cell cycle and apoptosis in healthy [21, 26, 35, 36, 45] and cancer $[6,13,28,30,33,34]$ cells suggests that these regulators could be mediators of curcumin action on ovarian cell functions. Viability, proliferation, and apoptosis of ovarian cells should affect their hormone release as mentioned above, although the opposite effect of steroid and peptide hormones on ovarian cell proliferation and apoptosis has also been documented [35, 38].

Curcumin action on ovarian cell mitosis and apoptosis can be mediated by its action on transcription factor p53, which stops the cell cycle, promotes DNA reparation, and induces apoptosis in defect or cancer ovarian cells [6]. On the other hand, there is evidence that p53 does not mediate the stimulatory action of curcumin on apoptosis in ovarian carcinoma cells. Evidence has shown that curcumin can activate proliferation promoter p38 mitogen-activated protein kinase (MAPK) and downregulate antiapoptotic peptides $\mathrm{Bcl}-2$ and survivin and Akt signaling [28]. The involvement of MAPK in mediating curcumin action has also been demonstrated for healthy ovarian cells [39].

The results of other studies have indicated that curcumin action on ovarian cancer cells or ovarian cells suffered from polycystic ovarian syndrome could be mediated by transcription factor STAT, matrix metalloproteinase-9 [6], sarco/endoplasmic reticulum Ca2+ ATPase [30], 5' AMNe-activated protein kinase, NF- $\kappa$ B, heat shock protein 70 [33,36], PI3K/Akt and Nrf2/HO-1 [21], AKT/mTOR/p70S6K [13], Wnt/ $\beta$-catenin signaling pathways [33], and enzymes SIRT-1 and SIRT-3 [44].

The antioxidant curcumin can bind reactive oxygen species and, therefore, prevent peroxidation and degradation of nucleic acids, peptides, lipids, and the consequent apoptosis, aging or malignant transformation of healthy cells, but not cancer cells [6, 
$19,44]$. It is not to be excluded that the protective effect of curcumin against pregnancy complications could also be due to its antioxidant properties [15]. Recently, the involvement of some microRNAs and long non-coding RNAs in regulating various types of cancer cells and curcumin action on these cells have been indicated $[13,33]$.

\section{Application in Reproductive Biology and Medicine}

The available reports regarding curcumin's effect on reproductive processes allows for outlining possible areas of its application. The ability of curcumin to promote ovarian follicullogenesis and fecundity in laboratory rodents [18, 19, 21,35-37] and rabbits [25] in vivo indicates its potential applicability as a natural biostimulator of reproduction in large farm animal production. Furthermore, its ability to improve oocyte maturation, quality, and developmental capacity $[19,36,37,45]$ suggests that its addition could be beneficial for in vitro oocyte maturation, fertilization, and embryo production.

Furthermore, evidence suggests its applicability for the prevention, mitigation, and maybe even treatment of some age-dependent reproductive disorders including late sexual maturation $[19,20]$, reproductive aging, ovarian insufficiency and failure [5, 20-22, 24, 36,37], complications during pregnancy [15], and a prolonged postpartum period [12]. Moreover, its potential to prevent, mitigate, and treat signs of ovarian cancer $[8,11,13,17,27-$ $34]$, polycystic ovarian syndrome $[35,43,44]$, and adverse effects of immunological shock [36], hypoxia [46], ionizing irradiation [45], ischemia [47], oxidative stress [44,48], mykotoxins [48], and arsenic [10] on ovarian functions suggests the large therapeutic potential of curcumin and its molecules.

It is, however, necessary to note that wider application of curcumin can be limited by its relatively low solubility, bioavailability, bioabsorption $[1,29,49]$, and stability in light and in organisms [34]. To improve these physicochemical and therapeutic characteristics, the following approaches have been tested:

1. Synthetic curcumin analogues with enhanced bioabsorption and a higher ability to block reactive oxygen species and cancer development [29].

2. Application of curcumin nanoparticles with higher absorption, transport through cell membranes and accumulation in the ovary [49], with increased stability and a proapoptotic effect on cancer cells [34], or with enhanced antiparasitic properties [16].

3. A combination of chemical modifications of curcumin and the generation of nanoparticles from the modified curcumin [32].

4. The application of lipid [9, 16,31] or metal [35] nanoparticles as carriers for curcumin transport.

The chemical modifications of curcumin and its carriers could expand the possibilities and efficiency of its application in animal production, biotechnology, and human and veterinary medicine.

\section{Conclusion and Possible Directions of Further Studies}

Rhizomes of Curcumas contain various biologically active substances, the best-known of which is polyphenol curcumin, which has a wide array of biological and medical effects. Its influence on female reproduction (puberty, reproductive aging, ovarian follicullogenesis and oogenesis, and fecundity) has been well documented. Curcumin can affect these processes via changes in the release and reception of pituitary and ovarian hormones, growth factors and cytokines. Furthermore, it can influence the response of ovarian cells to these substances and external environmental factors. Finally, curcumin can affect oxidative processes within the ovary and numerous intracellular signalling pathways related to ovarian cell proliferation and apoptosis. These effects described in the details above suggest the applicability of curcumin for stimulation of female reproductive processes in vivo and in vitro, as well as for the prevention, mitigation, and treatment of various reproductive disorders from ovarian insufficiency and infertility to polycystic ovarian syndrome and ovarian cancer.

On the other hand, numerous aspects of Curcumas' influence and application in the control of female reproduction require further studies. Previous investigations were focused mainly on curcumin, whilst reproductive effects of other constituents of Curcumas, including curcumin derivatives and curcuma oils, remain not studied as of yet. Hierarchical interrelationships between numerous mechanisms of curcumin action on reproduction require further elucidation. It could help to understand the causes of different actions of curcumin in vivo and in vitro on healthy cells and cancer cells. The available information concerning the effect and potential applicability of curcumin listed above was obtained mainly during animal and in vitro experiments, while clinical studies remain rare and insufficient. Increased bioavailability and efficiency of curcumin requires further efforts of specialists in chemistry and medicine. Nevertheless, the available information demonstrates that curcumin can be a promising, inexpensive, accessible, and efficient natural regulator, protector, and medicine for animal and human female reproductive processes.

\section{Contributors' Statement}

The manuscript has been generated by one author.

\section{Acknowledgements}

This work was funded by the Slovak Research and Development Agency (APVV) (projects no. APVV-15-0296) and by the Scientific Grant Agency of the Ministry of Education, Science, and Sport of Slovak Republic (VEGA) (project VEGA 13-ENV1321-02).

\section{Conflict of Interest}

The authors declare that they have no conflict of interest. 


\section{References}

[1] Lestari ML, Indrayanto G. Curcumin. Profiles Drug Subst Excip Relat Methodol 2014; 39: 113-204

[2] Kunnumakkara AB, Bordoloi D, Padmavathi G, Monisha J, Roy NK, Prasad S, Aggarwal BB. Curcumin, the golden nutraceutical: multitargeting for multiple chronic diseases. Br J Pharmacol 2017; 174: 1325-1348

[3] Dosoky NS, Setzer WN. Chemical composition and biological activities of essential oils of Curcuma species. Nutrients 2018; 10: 1196

[4] Ahmad RS, Hussain MB, Sultan MT, Arshad MS, Waheed M, Shariati MA, Plygun S, Hashempur MH. Biochemistry, safety, pharmacological activities, and clinical applications of turmeric: A mechanistic review. Evid Based Complement Alternat Med 2020; 2020: 7656919

[5] Tang C, Li L, Shi J, Wu D, Wang M, Wu Y, Yuan X. Curcumin in age-related diseases. Pharmazie 2020; 75: 534-539

[6] Mohebbati R, Anaeigoudari A, Khazdair MR. The effects of Curcuma longa and curcumin on reproductive systems. Endocr Regul 2017; 51: 220-228

[7] Tricco AC, Lillie E, Zarin W, O’Brien KK, Colquhoun H, Levac D, Moher D, Peters MDJ, Horsley T, Weeks L, Hempel S, Akl EA, Chang C, McGowan J, Stewart L, Hartling L, Aldcroft A, Wilson MG, Garritty C, Lewin S, Godfrey CM, Macdonald MT, Langlois EV, Soares-Weiser K, Moriarty J, Clifford T, Tunçalp Ö, Straus SE. PRISMA extension for Scoping Reviews (PRISMAScR): Checklist and explanation. Ann Intern Med 2018; 169: 467-473

[8] Vallianou NG, Evangelopoulos A, Schizas N, Kazazis C. Potential anticancer properties and mechanisms of action of curcumin. Anticancer Res 2015; 35: 645-651

[9] Nayak AP, Mills T, Norton I. Lipid based nanosystems for curcumin: past, present and future. Curr Pharm Des 2016; 22: 4247-4256

[10] Bahrami A, Sathyapalan T, Moallem SA, Sahebkar A. Counteracting arsenic toxicity: Curcumin to the rescue? J Hazard Mater 2020; 400: 123160

[11] Moody R, Wilson K, Jaworowski A, Plebanski M. Natural compounds with potential to modulate cancer therapies and self-reactive immune cells. Cancers (Basel) 2020; 12: 673

[12] Japheth KP, Kumaresan A, Patbandha TK, Baithalu RK, Selvan AS, Nag P, Manimaran A, Oberoi PS. Supplementation of a combination of herbs improves immunity, uterine cleansing and facilitate early resumption of ovarian cyclicity: A study on post-partum dairy buffaloes. J Ethnopharmacol 2021; 272: 113931

[13] Liu Y, Sun H, Makabel B, Cui Q, Li J, Su C, Ashby CR jr., Chen Z, Zhang J. The targeting of non-coding RNAs by curcumin: Facts and hopes for cancer therapy (Review). Oncol Rep 2019; 42: 20-34

[14] Momtazi AA, Derosa G, Maffioli P, Banach M, Sahebkar A. Role of microRNAs in the therapeutic effects of curcumin in non-cancer diseases. Mol Diagn Ther 2016; 20: 335-345

[15] Tossetta G, Fantone S, Giannubilo SR, Marzioni D. The multifaced actions of curcumin in pregnancy outcome. Antioxidants (Basel) 2021; 10: 126

[16] Rai M, Ingle AP, Pandit R, Paralikar P, Anasane N, Santos CAD. Curcumin and curcumin-loaded nanoparticles: antipathogenic and antiparasitic activities. Expert Rev Anti Infect Ther 2020; 18: 367-379

[17] Inano H, Onoda M, Inafuku N, Kubota M, Kamada Y, Osawa T, Kobayashi $\mathrm{H}$, Wakabayashi K. Potent preventive action of curcumin on radiation-induced initiation of mammary tumorigenesis in rats. Carcinogenesis 2000; 21: 1835-1841

[18] Murphy C], Tang H, Van Kirk EA, Shen Y, Murdoch W]. Reproductive effects of a pegylated curcumin. Reprod Toxicol 2012; 34: 120-124

[19] Azami SH, Nazarian H, Abdollahifar MA, Eini F, Farsani MA, Novin MG. The antioxidant curcumin postpones ovarian aging in young and middle-aged mice. Reprod Fertil Dev 2020; 32: 292-303
[20] Tiwari-Pandey R, Ram Sairam M. Modulation of ovarian structure and abdominal obesity in curcumin- and flutamide-treated aging FSH-R haploinsufficient mice. Reprod Sci 2009; 16: 539-550

[21] Yan Z, Dai Y, Fu H, Zheng Y, Bao D, Yin Y, Chen Q, Nie X, Hao Q, Hou D, Cui Y. Curcumin exerts a protective effect against premature ovarian failure in mice. J Mol Endocrinol 2018; 60: 261-271

[22] Melekoglu R, Ciftci O, Eraslan S, Cetin A, Basak N. Beneficial effects of curcumin and capsaicin on cyclophosphamide-induced premature ovarian failure in a rat model. J Ovarian Res 2018; 11: 33

[23] Koca SB, Yigit NO, Ozdogan HBE, Ozmen O. Reversible sterilization by supplementing turmeric (Curcuma longa) powder to diets of female Pseudotropheus socolofi. Fish Physiol Biochem 2020; 46: 1199-1205

[24] Destici Isgoren G, Dilbaz B, Erturk Aksakal S, Kiykac Altinbas S, Yildirim Z, Simsek G, Tapisiz OL. Impact of curcumin on ovarian reserve after tubal ligation: An experimental study. Reprod Sci 2021. doi:10.1007/s43032021-00468-8

[25] Sirotkin AV, Kadasi A, Stochmalova A, Balazi A, Földesiová M, Makovicky P, Chrenek P, Harrath AH. Effect of turmeric on the viability, ovarian folliculogenesis, fecundity, ovarian hormones and response to luteinizing hormone of rabbits. Animal 2018; 12: 1242-1249

[26] Kádasi A, Maruniaková N, Štochmalová A, Bauer M, Grossmann R, Harrath AH, Kolesárová A, Sirotkin AV. Direct effect of curcumin on porcine ovarian cell functions. Anim Reprod Sci 2017; 182: 77-83

[27] Shehzad A, Wahid F, Lee YS. Curcumin in cancer chemoprevention: molecular targets, pharmacokinetics, bioavailability, and clinical trials. Arch Pharm (Weinheim) 2010; 343: 489-499

[28] Watson JL, Greenshields A, Hill R, Hilchie A, Lee PW, Giacomantonio CA Hoskin DW. Curcumin-induced apoptosis in ovarian carcinoma cells is p53-independent and involves p38 mitogen-activated protein kinase activation and downregulation of $\mathrm{Bcl}-2$ and survivin expression and Akt signaling. Mol Carcinog 2010; 49: 13-24

[29] Rath KS, McCann GA, Cohn DE, Rivera BK, Kuppusamy P, Selvendiran K. Safe and targeted anticancer therapy for ovarian cancer using a novel class of curcumin analogs. J Ovarian Res 2013; 6: 35

[30] Seo JA, Kim B, Dhanasekaran DN, Tsang BK, Song YS. Curcumin induces apoptosis by inhibiting sarco/endoplasmic reticulum $\mathrm{Ca} 2+$ ATPase activity in ovarian cancer cells. Cancer Lett 2016; 371: 30-37

[31] Bondì ML, Emma MR, Botto C, Augello G, Azzolina A, Di Gaudio F, Craparo EF, Cavallaro G, Bachvarov D, Cervello M. Biocompatible lipid nanoparticles as carriers to improve curcumin efficacy in ovarian cancer treatment. J Agric Food Chem 2017; 65: 1342-1352

[32] Dwivedi P, Yuan S, Han S, Mangrio FA, Zhu Z, Lei F, Ming Z, Cheng L, Liu Z, Si T, Xu RX. Core-shell microencapsulation of curcumin in PLGA microparticles: programmed for application in ovarian cancer therapy. Artif Cells Nanomed Biotechnol 2018; 46 (sup3): S481-S491

[33] Hatamipour M, Ramezani M, Tabassi SAS, Johnston TP, Ramezani M, Sahebkar A. Demethoxycurcumin: A naturally occurring curcumin analogue with antitumor properties. J Cell Physiol 2018; 233: 9247-9260

[34] Duse L, Agel MR, Pinnapireddy SR, Schäfer J, Selo MA, Ehrhardt C, Bakowsky U. Photodynamic therapy of ovarian carcinoma cells with curcumin-loaded biodegradable polymeric nanoparticles. Pharmaceutics 2019; 11: 282

[35] Fatemi Abhari SM, Khanbabaei R, Hayati Roodbari N, Parivar K, Yaghmaei P. Curcumin-loaded super-paramagnetic iron oxide nanoparticle affects on apoptotic factors expression and histological changes in a prepubertal mouse model of polycystic ovary syndrome-induced by dehydroepiandrosterone - A molecular and stereological study. Life Sci 2020; 249: 117515

[36] Voznesens'ka Tlu, Bryzhina TM, Sukhina VS, Makohon NV, Aleksieieva IM. [Effect of NF-kappaB activation inhibitor curcumin on the oogenesis and follicular cell death in immune ovarian failure in mice]. Fiziol Zh 2010; 56: $96-101$ 
[37] Alekseyeva IN, Makogon NV, Bryzgina TM, Voznesenskaya TY, Sukhina VS. Effects of NF- $k B$ blocker curcumin on oogenesis and immunocompetent organ cells in immune ovarian injury in mice. Bull Exp Biol Med 2011; 151: 432-435

[38] Sirotkin AV. Regulators of ovarian Functions. Hauppauge, NY, USA: Nova Science Publishers, Inc.; 2014: 194

[39] Purwaningsih E, Soejono SK, Dasuki D, Meiyanto E. Curcumin inhibits luteal cell steroidogenesis by suppression of extracellular signal regulated kinase. Universa Medicina 2012; 31: 73-80

[40] Bachmeier BE, Mirisola V, Romeo F, Generoso L, Esposito A, Dell'eva R, Blengio F, Killian PH, Albini A, Pfeffer U. Reference profile correlation reveals estrogen-like transcriptional activity of Curcumin. Cell Physiol Biochem 2010; 26: 471-482

[41] Sirotkin AV, Harrath AH. Phytoestrogens and their effects. Eur J Pharmacol 2014; 741: 230-236

[42] Valentine SP, Le Nedelec MJ, Menzies AR, Scandlyn MJ, Goodin MG, Rosengren RJ. Curcumin modulates drug metabolizing enzymes in the female Swiss Webster mouse. Life Sci 2006; 78: 2391-2398

[43] Mohammadi S, Kayedpoor P, Karimzadeh-Bardei L, Nabiuni M. The effect of curcumin on TNF- $\alpha$, IL- 6 and CRP expression in a model of polycystic ovary syndrome as an inflammation state. J Reprod Infertil 2017; 18: $352-360$
[44] Heshmati J, Golab F, Morvaridzadeh M, Potter E, Akbari-Fakhrabadi M, Farsi F, Tanbakooei S, Shidfar F. The effects of curcumin supplementation on oxidative stress, Sirtuin-1 and peroxisome proliferator activated receptor $\gamma$ coactivator $1 \alpha$ gene expression in polycystic ovarian syndrome (PCOS) patients: A randomized placebo-controlled clinical trial. Diabetes Metab Syndr 2020; 14: 77-82

[45] Aktas C, Kanter M, Kocak Z. Antiapoptotic and proliferative activity of curcumin on ovarian follicles in mice exposed to whole body ionizing radiation. Toxicol Ind Health 2012; 28: 852-863

[46] Sak ME, Soydinc HE, Sak S, Evsen MS, Alabalik U, Akdemir F, Gul T. The protective effect of curcumin on ischemia-reperfusion injury in rat ovary. Int J Surg 2013; 11: 967-970

[47] Eser A, Hizli D, Haltas H, Namuslu M, Kosus A, Kosus N, Kafali H. Effects of curcumin on ovarian ischemia-reperfusion injury in a rat model. Biomed Rep 2015; 3: 807-813

[48] Qin X, Cao M, Lai F, Yang F, Ge W, Zhang X, Cheng S, Sun X, Qin G, Shen W, Li L. Oxidative stress induced by zearalenone in porcine granulosa cells and its rescue by curcumin in vitro. PLoS One 2015; 10: e0127551

[49] Arozal W, Ramadanty WT, Louisa M, Satyana RPU, Hartono G, Fatrin S, Purbadi S, Estuningtyas A, Instiaty I. Pharmacokinetic profile of curcumin and nanocurcumin in plasma, ovary, and other tissues. Drug Res (Stuttg) 2019; 69: 559-564 